\title{
Experimental Analysis of OLSR and DSDV Protocols on NS-2.35 in Mobile Ad-Hoc Networks
}

\author{
Shelja Sharma \\ Research Scholar, Department of Computer Science \& Engineering, Faculty of Engineering \& Technology, Manav \\ Rachna International University, Faridabad, Haryana, India \\ Email: shelja.fet@mriu.edu.in \\ Suresh Kumar Bhardwaj \\ Department of Computer Science \& Engineering, Faculty of Engineering \& Technology, Manav Rachna International \\ University, Faridabad, Haryana, India \\ Email: suresh.fet@mriu.edu.in
}

\begin{abstract}
Mobile ad-hoc networks are autonomous networks of intelligent mobile nodes. Designing an efficient routing protocol with goal efficient route establishment is still a burning research issue. Routing protocols are broadly classified into proactive and reactive protocols on the basis of their nature of working. In this paper, we present comparative simulation analysis of two proactive protocols namely, Optimized Link State Routing (OLSR) and Destination-Sequenced Distance Vector (DSDV). Simulations have been carried out multiple times using Network Simulator-2.35 (NS-2.35), on random scenario patterns and compared in two environments (OLSR and DSDV) by varying the network size and mobility of nodes. We have patched and installed OLSR protocol on NS-2.35 as it's not available as a part of NS-2.35 installation. The simulation results indicate that, OLSR perform better than DSDV protocol for application oriented metrices such as packet delivery fraction, packet loss and end-to-end delay. But, Routing Overheads is significantly consistently higher for OLSR protocol in all the cases considered, which is proven experimentally. Furthermore, based upon the simulation results, work can be done in the direction of improving the performance of the OLSR protocol to make it a choice of proactive protocol for large and denser network.
\end{abstract}

Index Terms-Mobile Ad Hoc Networks, Routing Protocols, Optimized Link State Routing Protocol, Destination Sequenced Distance Vector Routing Protocol.

\section{INTRODUCTION}

Mobile ad-hoc networks (MANETs) are independent, instantaneous \& short live networks of autonomous mobile nodes. Being compelled by nature, nodes can move randomly and reorganizes themselves into an arbitrary network as shown in Figure 1. Each node in MANET must act as a router in order to enable multi-hop radio relaying. Mobile ad-hoc network is an emerging research area because of its widespread utility and easy establishment. These wireless networks offers reduced prices, improved flexibility \& higher speed and have fascinated significant interest in current years, enjoying continues rapid growth.

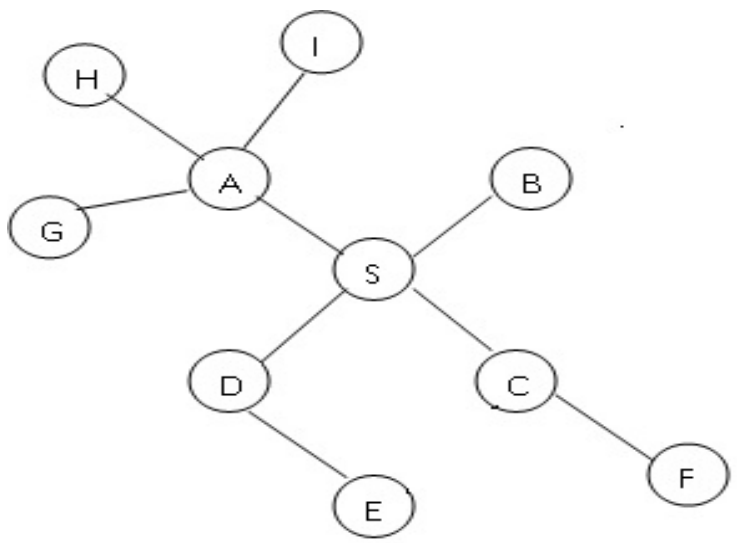

Fig.1. Example of Mobile Ad Hoc Network

There are several key issues in the design of an efficient mobile ad-hoc network. One of the hot burning issues is developing an efficient routing protocol, which compromises on resource constraints and offer goal efficient route establishment between two entities. The ad-hoc routing protocols are broadly classified into proactive and reactive protocols on the basis of their nature of routing information update mechanism. As per the working nature of proactive or table driven routing protocols, each node maintains route to every other node in the network, prior to data transmission and irrespective of the necessity of the route. As an example, primary fields maintained in a route table by a particular node in Destination Sequenced Distance Vector (DSDV) Proactive Routing Protocol are shown in Table 1. Where, 'Destination' represents the address of a destination node, 'Next Hop' represents the address of a next node along the route towards destination node and 'Hop Count' 
represents the number of nodes required to reach the destination node.

Table 1. Example of Routing Table in DSDV Proactive Protocol

\begin{tabular}{|c|l|l|}
\hline Destination & Next Hop & Hop Count \\
\hline A & A & 0 \\
\hline B & S & 2 \\
\hline C & S & 2 \\
\hline D & S & 2 \\
\hline E & S & 3 \\
\hline F & S & 3 \\
\hline G & G & 1 \\
\hline H & H & 1 \\
\hline I & I & 1 \\
\hline S & S & 1 \\
\hline
\end{tabular}

On the contrary, in reactive or on-demand routing protocols, each node discovers route to desired destination only on demand basis by flooding route request packet as shown in Figure 2.

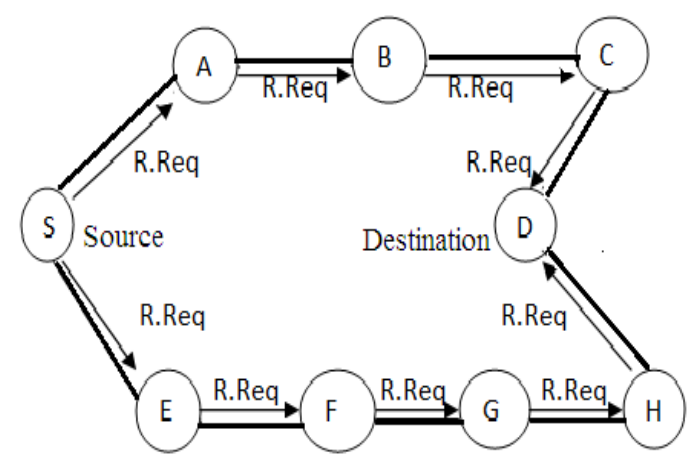

Fig.2. Propagation of Route Request Packet in On-Demand Protocols

The Destination Sequenced Distance Vector (DSDV) routing protocol periodically exchange routing information in order to constantly keep track of routing information, maintained in the routing table. Every node in the network is engaged in propagating periodic or event triggered routing updates. Likewise, Optimized Link State Routing (OLSR) protocol is proactive by nature and each node records \& maintains link status information to all other neighbor nodes but only some selected nodes, called Multi-point relay nodes are eligible to propagate control information.

As per the authors in references $[1,2]$, the development of OLSR protocol was an attempt to reduce control overhead as it does not generate extra control traffic in response to link failures and link additions unlike previous proactive protocols like DSDV and it employs the concept of multipoint relay nodes to utilize only subset of neighbor links for forwarding the control information. So, only some nodes are engaged in forwarding control information in contrast to DSDV protocol $[3,4]$ in which all the nodes participate in forwarding routing information.

In this paper, we aimed at comparing the relative performance of these two proactive routing protocols namely, OLSR and DSDV. For simulation and analysis, Network Simulator-2.35 (NS-2.35) is chosen, which is an Open Source and freely available widely for networking research community. In contrast to DSDV protocol, OLSR protocol is not available as a part of NS-2.35 installation therefore; we have patched and installed OLSR protocol on network simulator- 2.35. Simulations have been carried out multiple times, on random scenario patterns and compared in two environments (OLSR and DSDV) by varying the network size and mobility of nodes. The four performance metrics namely, packet delivery fraction, routing overhead, packet loss and endto-end delay are considered for evaluating the performance of the two protocols. After various simulation runs, average value of the results has been considered for evaluating performance metrics and plotting graphs.

Theoretically it has been said that [1, 2], OLSR reduces routing overheads but practically simulation results indicate that, OLSR protocol performs better as compared to DSDV protocol for all the cases considered, but acquire consistently higher control overheads irrespective of varying network load and mobility of nodes. Consequently, in future work can be done in the direction of reducing control overheads in OLSR protocol to make it a choice of proactive protocol for large scale mobile networks.

\section{LITERATURE REVIEW}

P. Jacquet $[1,2]$ et al., have proposed and discussed an Optimized Link State Routing Protocol for wireless Networks. The key concept used in the protocol is that of multipoint relays (MPRs). The MPRs are some of the selected nodes which forward broadcast messages during the flooding process. As per the Authors, this technique substantially reduces the message overhead as compared to a classical flooding mechanism, where every node retransmits each message when it receives the first copy of the message.

E. Mahdipour [5] et al., have evaluated the performance of DSDV routing Protocol through simulations using NS2 simulator. They carried out simulations with Constant Bit Rate (CBR) traffic pattern by varying network load and pause time, to calculate percentage of the dropped packets and end to end delay. As per the simulations results, Authors have reported the increase in drop ratio as the node speed increases and increase in end-to-end delay if no. of connections also increases with increase in node speed.

Zheng Yihui [6] et al., have modified the Multipoint Relay selection criteria in OLSR protocol. They have proposed Node-Status Self-Sensing Optimized Link-State Routing Protocol (N3S-OLSR), which uses self-sensing information maintained by each node and hop count metric, to optimize network performance. Each node maintains self sensing information, which includes Node Status Information (Mobile Node's Current Location or Current Residual Energy value or CPU Occupancy Rate) and Link Status Information (Link Signal Strength or 
Link Delay or Distance). They have concluded that, N3SOLSR, offer more stable path than OLSR protocol, as it avoids choosing the neighbors with low residual energy as MPR nodes.

Deepali Arora [7] et al,, have performed the performance assessment of AODV, DYMO and OLSR protocols for large scale denser MANETs. They have performed simulations by varying node velocity using OMNET++ simulator. Based on the simulation results authors claimed that, they are the first to report that, OLSR protocol does not perform well in large-scale, denser multi-hop dynamic network, except when majority of nodes are nearly stationary and their results contradicts prior claims in references $[1,8,9]$ that, OLSR is particularly suitable for large scale denser network. Similarly, S. Mohapatra and P. Kanungo [8] concluded that, if terrain size is more than $600 * 600 m$ OLSR performs well in terms of packet delivery fraction for high mobility conditions.

S. Mohapatra and P. Kanungo [8], have performed performance analysis of AODV, DSR, OLSR and DSDV protocols using NS2 simulator by varying simulation area, number of nodes and pause time. As per the simulation results, authors concluded that, if terrain size is more than $600 \mathrm{~m} * 600 \mathrm{~m}$ and if packet delivery ratio and throughput are the prime criteria then, the OLSR protocol is better solution for high mobility conditions.

Shakeera [19], has reported the redundancy in the MPR selection algorithm in the OLSR protocol. Author has augmented the optimization scheme to OLSR Protocol and proposed an algorithm called "Necessity First Algorithm (NFA)" to select the Optimal Multipoint Relays in OLSR protocol in order to improve the performance of the greedy algorithm.

Yamada Kenji [21] et al., have reported that, redundancy is caused in MPR selection procedure of OLSR protocol, which run on each node independently of its neighbor nodes. They have proposed, cooperative MPR selection procedure, to reduce number of routing packets in high-density network. As per their proposal, redundant control messages can be piggybacked with other control messages in dense networks to reduce control traffic in the network.

Shahram Behzad [23] et al., have proposed improvement in OLSR routing protocol by eliminating the unnecessary loops. They have performed simulations on NS-2.35 simulator by varying number of nodes. Their proposed OLSR protocol improved throughput and packet delivery fraction by $20 \%$ as compared to conventional OLSR protocol.

\section{OVERVIEW OF DSDV AND OLSR PROTOCOLS}

\section{A. Destination Sequenced Distance Vector (DSDV) Protocol}

The DSDV protocol is a table driven routing scheme. Being compelled by proactive nature of the protocol, each node relies on periodic and/or event triggered routing updates in order to maintain consistent topology information throughout the network. Therefore, all the nodes in the network are engaged in control message propagation irrespective of data transmission as shown in Figure 3. Each node is engaged in the following three activities:

- Route table Creation: Each node maintains routing table with entry for route to every other node in the network. Route table entries are: <destination address, destintion_sequence_no, next-hop, hop count, install time>.

- Route table Advertisement: Every time on advertising the particular node increases it's sequence no. by 2(evenly) and in case of broken links, node increases sequence no. by 1(changed to odd no.) and set hop count (metric) to infinity. Route advertisement message includes the entries for: <destination address, hop count, destintion_sequence_no $>$.

- Routing Update: Each node transmits periodic and/or event triggered routing updates to keep table consistency. Routing update entries include: <destination address, destintion_sequence_no, hopcount $>$.

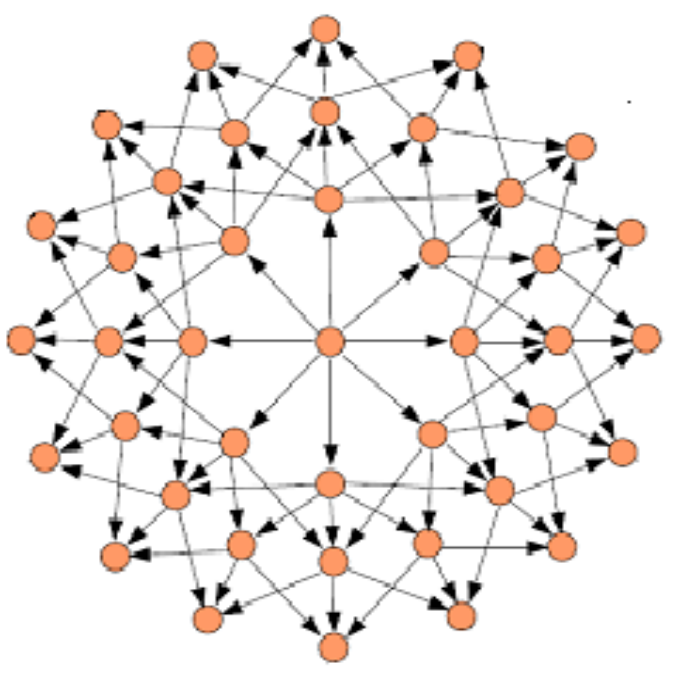

Fig.3. Control Message Propagation in DSDV Protocol

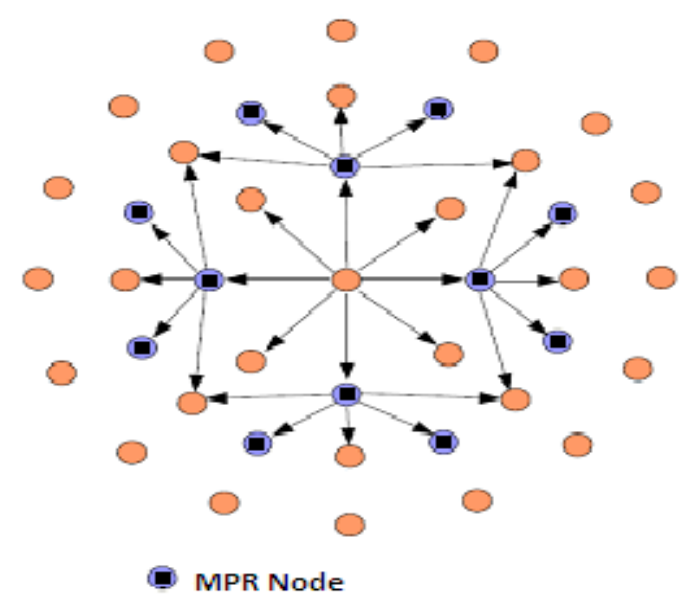

Fig.4. MPR Flooding in OLSR Protocol 


\section{B. Optimized Link State Routing( OLSR) Protocol}

The Optimized Link State Routing is also a proactive protocol in mobile ad-hoc networks, which maintains link status information. The protocol employs the key concept of "Multipoint Relays" (MPRs). Some 1-hop neighbor nodes are selected as Multipoint Relay (MPR) nodes, to generate and forward topology control (TC) messages throughout the network as shown in Figure 4. Thus it should reduce the overall network traffic to a large extent as compared to other proactive protocols.

Working of the OLSR protocol can be aggregated in the following steps:

- Neighbor Sensing through Hello Message Broadcast.

- Hello Message Processing and Construction of Neighbor Table.

- Execution of MPR Selection Algorithm at Each Node.

- Construction of MPR Selector table.

- Topology Control message Broadcast or Multipoint Relay Information Declaration.

- Topology Control Message processing and Construction of Duplicate Table.

- Construction of Topology Table through Topology Control Message.

- Construction of Routing Table.

In order to obtain the link status information throughout the network, the OLSR protocol uses two types of messages viz. hello message and topology control (TC) messages. Individual nodes calculate route to all other nodes through multipoint relay nodes, based on the topology information obtained from TC messages.

\section{Simulation ENVIORNMENT/ SETUP}

For simulation and analysis, we have used NS-2.35 discrete event simulator, which was developed by Monarch Research Group in Carnegie Mellon University [10]. The AODV, DSDV and DSR protocols are provided as part of the NS-2 installation but, OLSR protocol is not available as a part of NS-2.35. We have taken third party software, developed by university of Murcia, Spain [11] called UM-OLSR, which is an implementation of OLSR protocol for NS-2 simulator. We have used "um-olsr1.0.tgz" for patching \& installing OLSR protocol in NS2.35. We have generated thirty random scenario patterns, by varying pause time and number of nodes and five Constant Bit Rate (CBR) traffic files, by varying the number of nodes. To simulate any network on NS2, we have to specify and assign the network parameters for simulation. This is done by configuring the simulator with the simulation parameters namely, the type of traffic pattern, protocol used, number of nodes, mobility model, simulation time, terrain size etc. as shown in Table 2.
Table 2. Simulation Parameters

\begin{tabular}{|l|l|l|}
\hline S. No & Parameters & Value \\
\hline 1 & Routing Protocols & DSDV and OLSR \\
\hline 2 & MAC Layer & IEEE $802.11 \mathrm{~b}$ \\
\hline 3 & Simulation Time & $200 \mathrm{~s}$ \\
\hline 4 & Terrain Size & $800 \mathrm{~m} \times 500 \mathrm{~m}$ \\
\hline 5 & Number of Nodes & $15-20-25-30-35$ \\
\hline 6 & Mobility Model & Random Waypoint \\
\hline 7 & Traffic Type & CBR \\
\hline 8 & Pause time & $0 \mathrm{~s}-200 \mathrm{~s}($ interval-50s $)$ \\
\hline 9 & Speed & $20 \mathrm{~m} / \mathrm{s}$ \\
\hline 10 & Node Placement & Random \\
\hline 11 & Number of connections & 10 \\
\hline 12 & Packet size & 512 bytes \\
\hline 13 & Packet Send Rate & 4 packets/sec \\
\hline
\end{tabular}

Each run of the simulator accepts a scenario file as input, which describes the position and motion of each node and the sequence of packets originating from each node. The detailed trace files created by each run are stored on disk, and analyzed using a script-routine (written in awk script), that counts the number of packets successfully delivered and the length of the paths taken by the packets, as well as additional information about the internal functioning of each protocol. The NS2 simulator gives two files as output; NAM (Network Animator) generates NAM file, which is used for graphical visualization and other file called trace file is used for calculating the results. A sample screen -shot of NAM is shown in Figure 5.

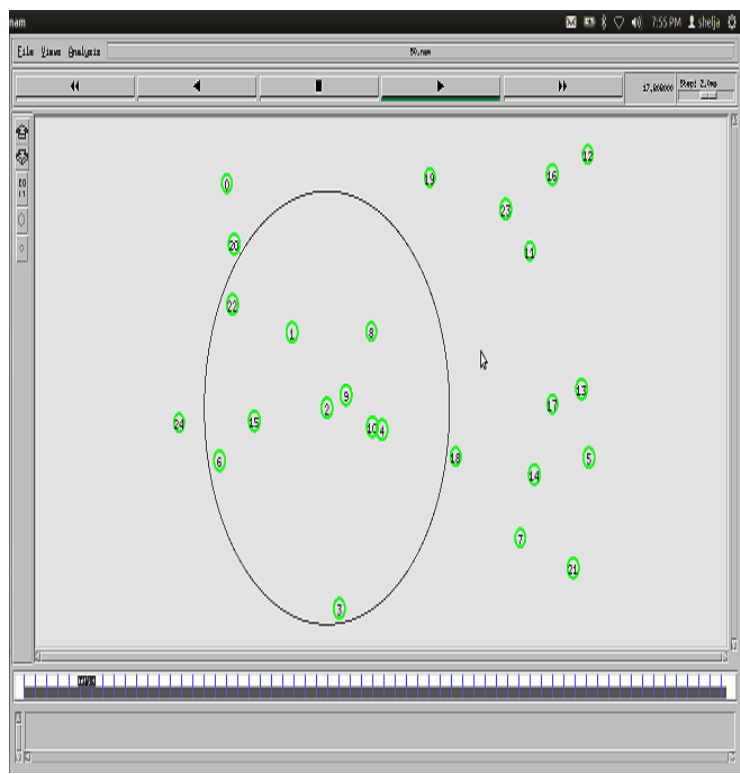

Fig.5. Sample Snapshot taken during the Simulation Process

There are various performance metrics for comparing and evaluating the effectiveness of various routing protocols as given below:-

Throughput: It is the ratio of the total number of packets received by the destination nodes over total number of packets sent by the source node. 
Average End-to-End Delay: It represents the total time taken by a packet to travel from the source node to the destination node. It includes all possible delays on the path.

Routing Overheads: All the routing control packets transmitted during simulation in order to discover and maintain routes from source to destination nodes.

Normalized Routing Load (NRL): It is the measure of number of routing packets transmitted for each data packet delivered at the destination node.

Packet Delivery Fraction (PDF): It is the ratio of the total number of data packets received by the destination nodes over total number of data packets sent by the source nodes.

Packets Loss: It is the measure of number of data packets, failed to reach the destinations or number of packets dropped by routers due to various reasons.

Simulations have been carried out multiple times, by varying mobility of nodes and network size, on random scenario patterns. The average value of the results has been considered for evaluating the performance metrics namely, Packet Delivery Fraction, Routing Overhead, Packet Loss and End-to-End delay. Two cases have been considered, in case I, network load analysis is done by varying number of nodes from 15 to 35 nodes as given in Table 2 and pause time is kept constant as 100 (s). Whereas in case II, we have done mobility analysis, by varying pause time from 0 (s) to 200 (s) in an interval of 50 (s) as given in Table 2 and number of nodes have been kept constant as 25 nodes.

\section{Simulation Analysis And Results}

To investigate the network performance, this section presents various simulation results and their analysis in the two cases considered.

\section{A. Case I: Network Load Analysis-Analyzing effect of varying network size}

In this analysis, numbers of nodes have been varied from 15 to 35 with an increment of 5 nodes whereas pause time, terrain size and simulation time are fixed at $100 \mathrm{~s}, 800 \mathrm{~m} \times 500 \mathrm{~m}$ and 200 s respectively. Other network parameters are same as described in Table 1. In simulation, 10 random scenarios and 5 traffic files are generated. The average value of results obtained from multiple simulations run is used to plot the performance of the protocols by varying the number of nodes.

Packet Delivery Fraction: The Packet Delivery Fraction (PDF) of the two protocols is shown in Figure 6. It is observed that PDF of the OLSR protocol is higher than DSDV protocol at all points. Considering, individual fall/increase, as the traffic sources increases, there is a slight fall in PDF of OLSR protocol at 25 and 30 nodes and slight increase in PDF of DSDV protocol at 30 nodes. Reason behind is, as the network load increases number of Topology Control (TC) messages and Hello messages increases in the network in order to select and maintain Multipoint Relay (MPR) nodes and it increases congestion in the network.

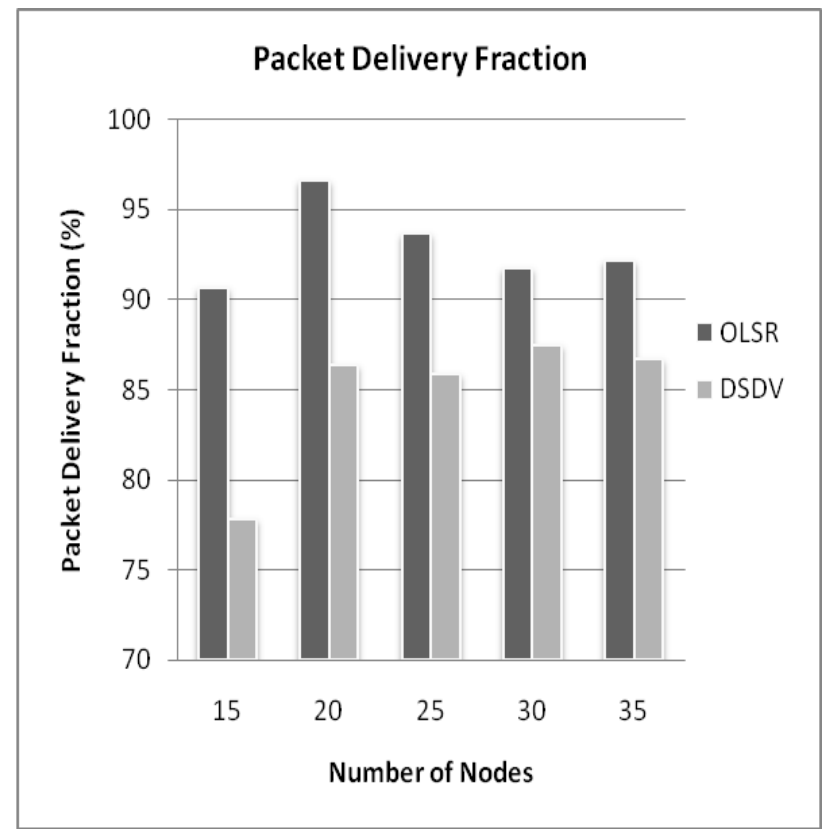

Fig.6. Packet Delivery Fraction vs. Number of Nodes

Routing Overheads: It is observed from the Figure 7 that, the Routing Overheads of OLSR Protocol is higher than DSDV protocol for all the cases. The reason behind is that, control messages are transmitted more frequently in OLSR protocol as compared to the DSDV protocol. In OLSR protocol, the default TC message transmission interval is set to 5 second and the default Hello message transmission interval is set to 2 second, whereas DSDV periodic route update interval is set to 15 second by default.

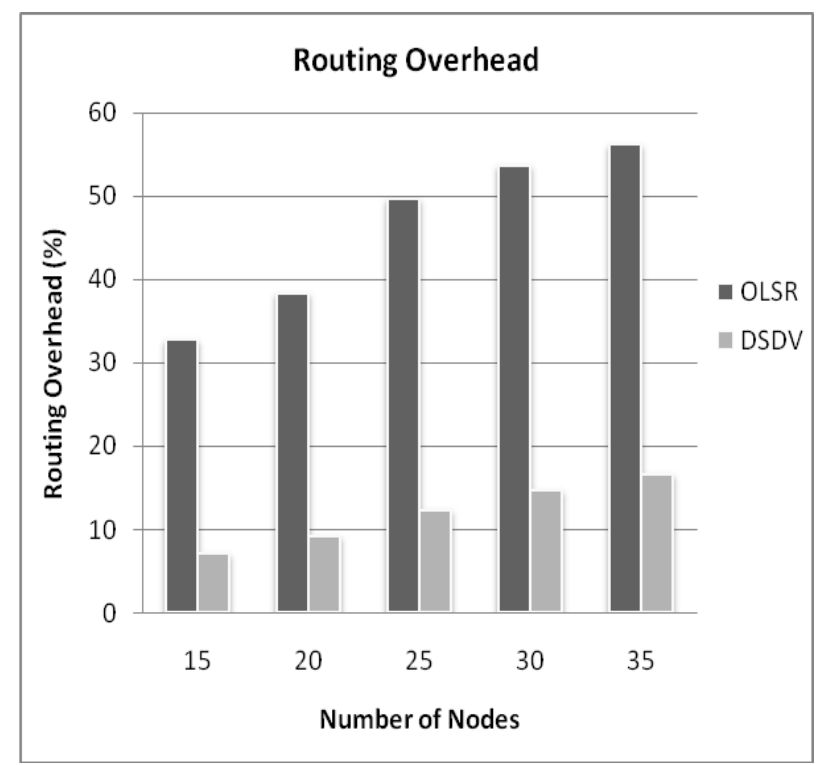

Fig.7. Routing Overhead vs. Number of Nodes

Packet Loss: It is the measure of number of packets successfully delivered to the destination nodes. The number of dropped packets for the two protocols, as a function of the number of nodes is shown in Figure 8. The dropped packet rate of DSDV protocol is comparatively higher than OLSR protocol, as OLSR 
offers more stable routes through MPR nodes, as compared to DSDV protocol and the DSDV protocol relies on stale route entries in the absence of fresh up-todate routes.

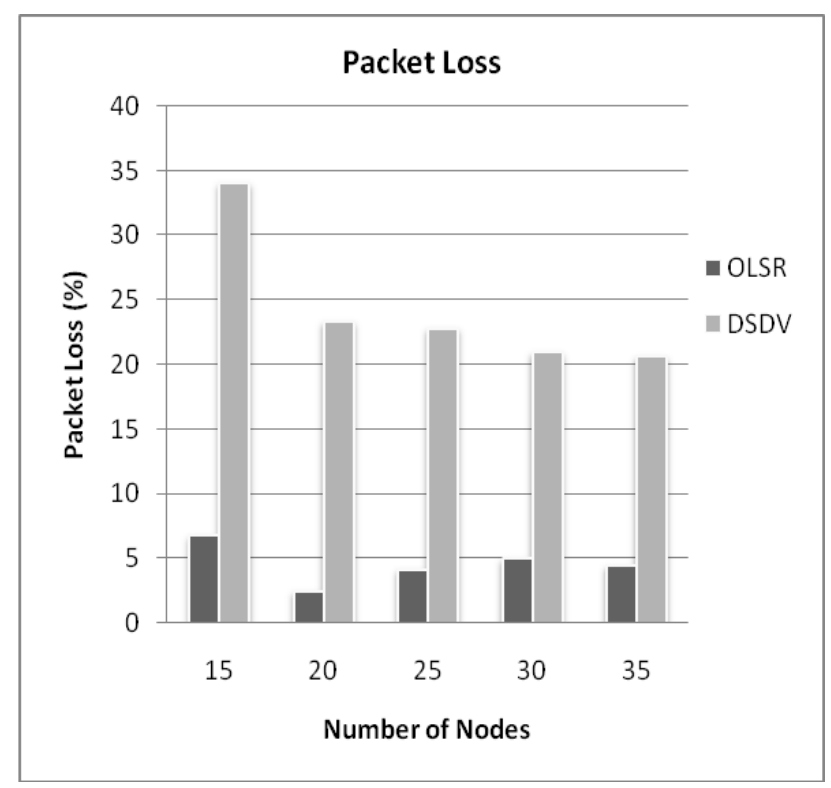

Fig.8. Packet Loss vs. Number of Nodes

End-to-End Delay: Figure 9 depicts the End-to-End Delay of the DSDV and OLSR protocols. It includes all delays experienced by a packet to travel from the source node to the corresponding destination node. The end-toend delay of DSDV protocol is slightly higher than OLSR protocol at some points as shown in Figure 9. The reason behind is, DSDV protocol uses stale routes in the absence of fresh routes whereas path offered through MPR nodes in OLSR protocol is more reliable \& stable. Otherwise, as per the simulation results there is not a significant difference in End-to-End Delay of both protocols when network load is varied.

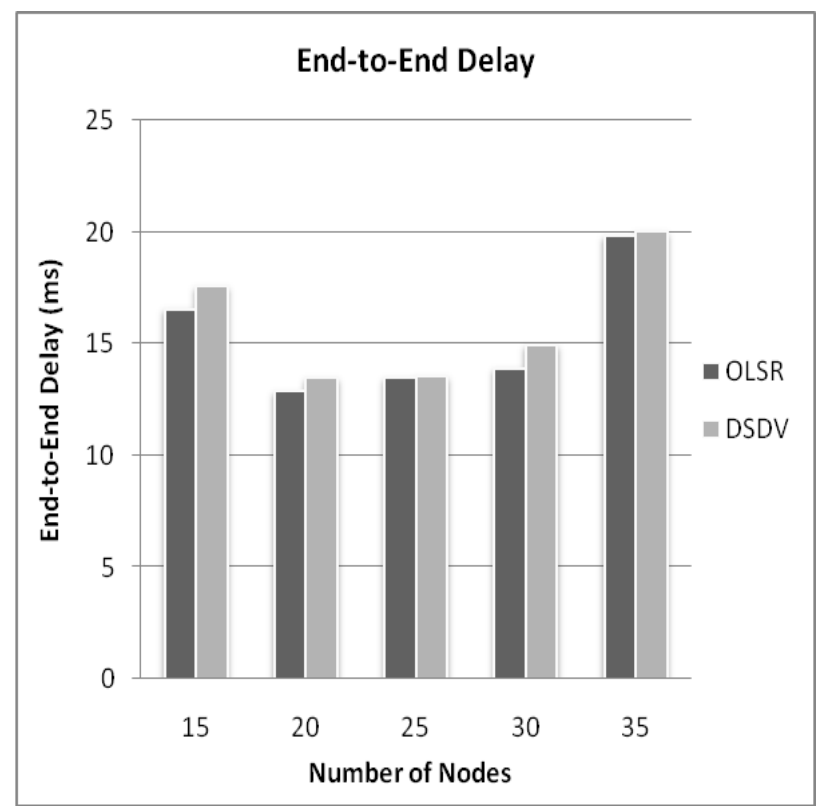

Fig.9. End-to-End Delay vs. Number of nodes
B. Case II: Mobility Analysis: Analyzing effect of varying mobility of nodes

In order to analyze the effect of varying mobility on the performance of the protocols, the pause time has been varied from $0(\mathrm{~s})$ to $200(\mathrm{~s})$ in an interval of $50(\mathrm{~s})$. Whereas number of nodes, terrain size and simulation time are fixed at 25 nodes, $800 \mathrm{~m} \times 500 \mathrm{~m}$ and $200 \mathrm{~s}$ respectively. The Constant Bit Rate traffic type is used with 512 byte data packet size. Rate of packet transmission is 4packets/second. Other parameters remain the same as given in Table 2.

Packet Delivery Fraction: The Packet Delivery Fraction (PDF) of both the protocols is shown in Figure 10. It is observed that, individually PDF of the two protocols is varying continuously. The PDF of OLSR protocol is higher than DSDV protocol at all points. The reason behind is, OLSR protocol offers more stable path in mobile networks through MPR nodes, whereas DSDV relies on periodic/event triggered routing updates for broken link repair and it uses stale routes in the absence of fresh and up-to-date routes in high mobility scenarios.

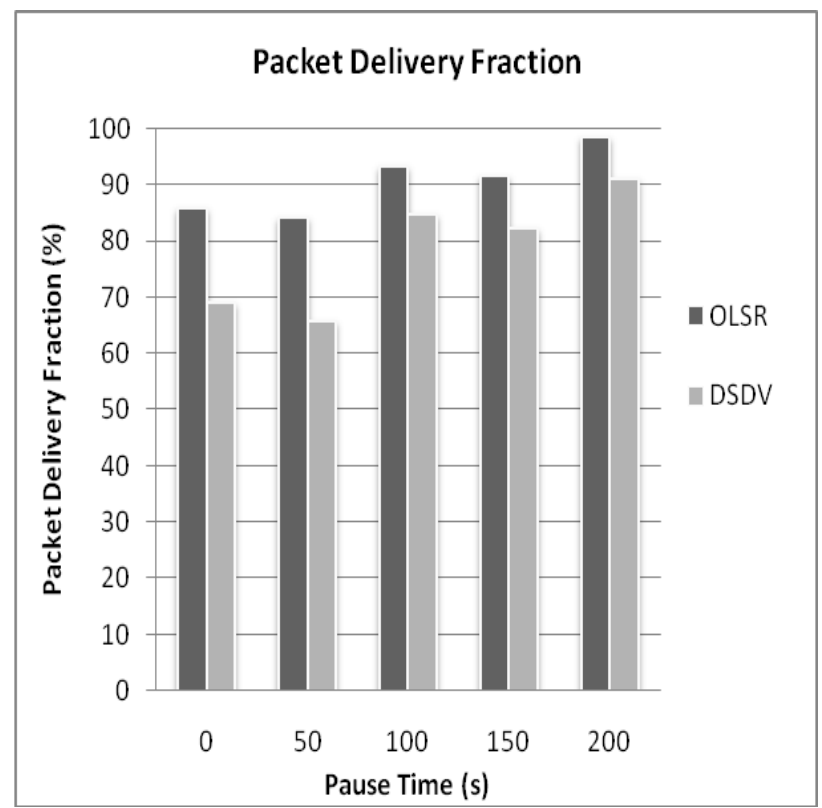

Fig.10. Packet Delivery Fraction vs. Pause Time

Routing Overheads: It is observed from Figure 11 that, the Routing Overheads of OLSR Protocol is higher than DSDV protocol for all the scenarios irrespective of mobility of nodes. Theoretically, the OLSR protocol should bear less control overheads as compared to the DSDV protocol. But, the control messages are transmitted more frequently in OLSR protocol as compared to the DSDV protocol. In OLSR protocol the default Transmission Control message transmission interval is set to 5 second and the default Hello message transmission interval is set to 2 second, whereas DSDV periodic route update interval is set to 15 second by default. Therefore, default control message transmission intervals can be considered as one of the reason for consistently higher Routing Overheads in OLSR protocol as compared to the DSDV protocol. 


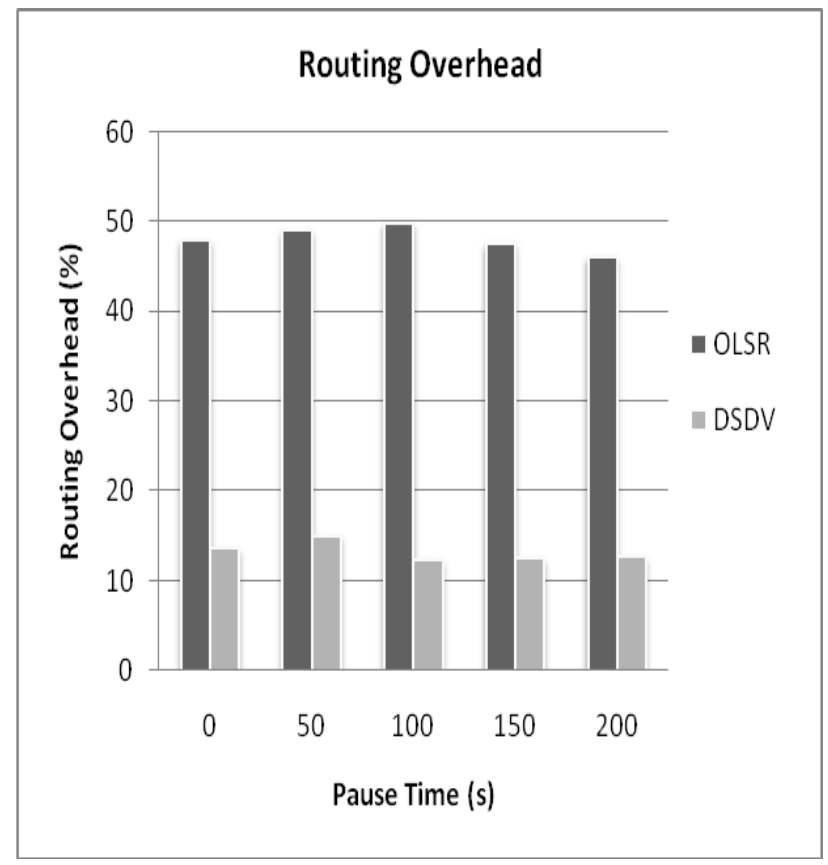

Fig.11. Routing Overhead vs. Pause Time

Packet Loss: It is observed that, the dropped packet rate of DSDV protocol is comparatively higher than the OLSR protocol as shown in Figure 12. When nodes are continuously moving at pause time $0(\mathrm{~s})$ and $50(\mathrm{~s})$, the Packet loss of DSDV protocol is much higher than the OLSR protocol, because OLSR offers more stable routes through MPR nodes as compared to the DSDV protocol which relies on stale routes in high mobility scenarios. Therefore, in terms of Packet Loss, performance of the OLSR protocol is better than the DSDV protocol.

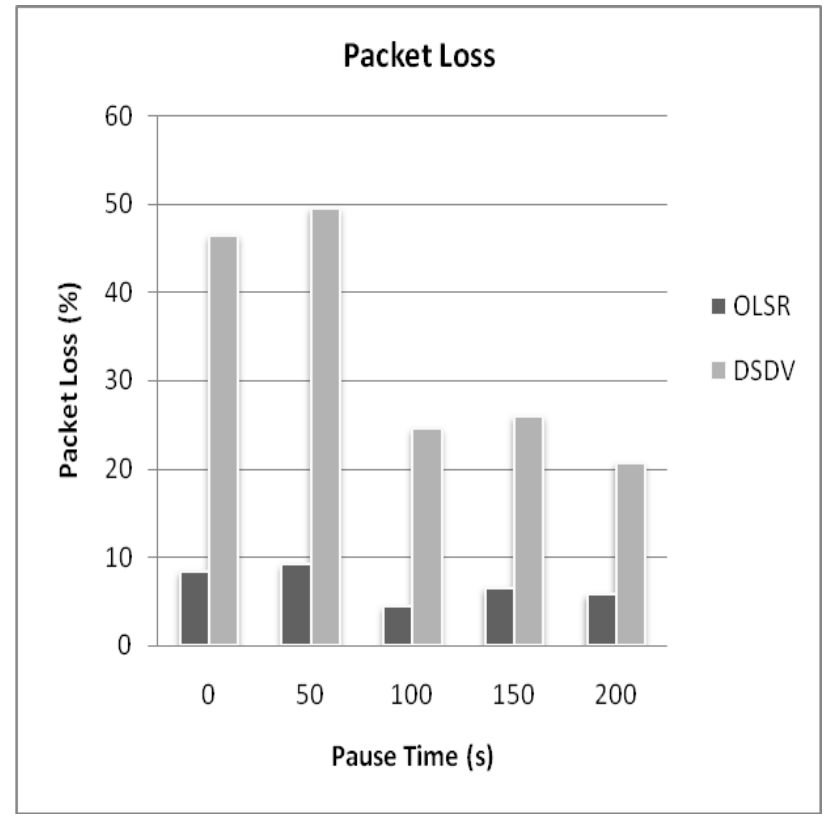

Fig.12. Packet Loss vs. Pause Time

End-to-End Delay: The End-End Delay is varying continuously for both protocols as shown in Figure 13. When nodes are continuously moving at pause time $0(\mathrm{~s})$, the End-to-End Delay of DSDV protocol is higher than the OLSR protocol, as it uses stale routes in the absence of fresh and up-to-date routes.

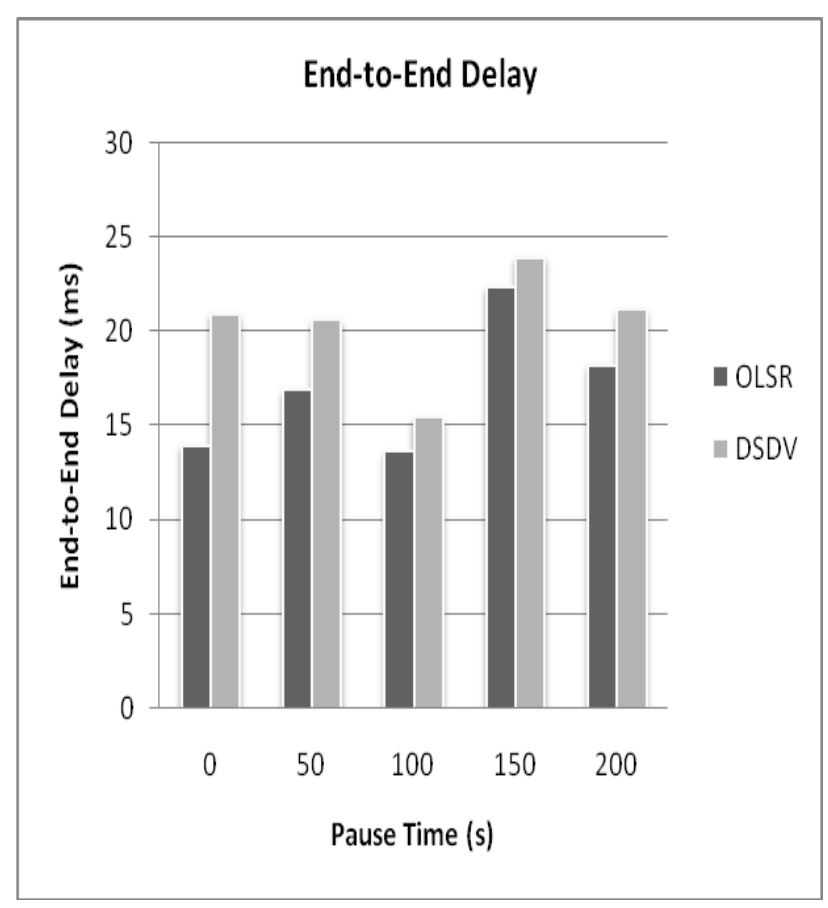

Fig.13. End-End Delay vs. Pause Time

\section{CONCLUSION}

Destination Sequenced Distance Vector (DSDV) and Optimized Link State Routing (OLSR), both employ proactive routing approach but with different routing scheme. In this paper, we have performed simulation analysis of DSDV and OLSR protocols on NS-2.35 simulator. As per the literature, the concept of MPR Nodes in the OLSR protocol was introduced in order to reduce the routing overheads of proactive protocols. Theoretically, this concept is alright but, experimentally it did not perform well. One of the reasons for consistently higher control overheads in OLSR protocol is, comparatively small control message transmission interval (by default) i.e. control messages are transmitted more frequently in OLSR protocol as compared to the DSDV protocol.

As per the simulation results, the general observation is that for application oriented metrics such as packet delivery fraction, packet loss and end-end delay, the OLSR protocol outperforms DSDV protocol in highly mobile network. But, routing overheads in the OLSR protocol is significantly higher for all scenarios, irrespective of change in network load or mobility of nodes. However, it is observed from the simulation results that, the effect of higher control overheads in OLSR protocol is counteracted by relatively higher packet delivery fraction. Based on experimental and theoretical analysis, comparison of DSDV and OLSR Protocols is presented in Table 3.

Further, work can be done to reduce the routing overheads of OLSR protocol by employing different 
routing scheme, which can make it more suitable for highly dense and mobile networks unlike DSDV protocol. Therefore, our future work includes performance optimization of OLSR protocol by making an attempt to reduce the routing overheads.

Table 3. Comparative Analysis of DSDV and OLSR Routing Protocols

\begin{tabular}{|c|c|c|}
\hline Parameters & DSDV & OLSR \\
\hline $\begin{array}{l}\text { Packet Delivery } \\
\text { Fraction }\end{array}$ & Lower & Higher \\
\hline Routing Overhead & Lower & Higher \\
\hline Packet Loss & Higher & Lower \\
\hline End-to-End Delay & Higher & Lower \\
\hline $\begin{array}{l}\text { Number of Nodes } \\
\text { participating in } \\
\text { Message propagation }\end{array}$ & All & $\begin{array}{l}\text { Only } \\
\text { nodes }\end{array}$ \\
\hline $\begin{array}{l}\text { Communication } \\
\text { Overhead }\end{array}$ & Higher & Lower \\
\hline Sleep mode Operation & Absent & Present \\
\hline $\begin{array}{l}\text { Process of Route } \\
\text { Construction }\end{array}$ & Simpler and Easier & $\begin{array}{l}\text { Complex, } \\
\text { Cumbersome } \\
\text { and Time- } \\
\text { Consuming }\end{array}$ \\
\hline Memory Overhead & Lower & Higher \\
\hline
\end{tabular}

\section{REFERENCES}

[1] Jacquet P., Muhlethaler P., Clausen T., Laouiti A, Qayyum A and Viennot L., "Optimized link state routing protocol for ad hoc networks", In proceedings of the 5th IEEE Multi Topic Conference INMIC, 2001, pp. 62-68.

[2] Clausen T. and Jacquet P., "Optimized Link State Routing Protocol (OLSR)", RFC 3626, IETF Network Working Group, available online at https://www.ietf.org/ rfc/rfc3626.txt, October 2003.

[3] Perkins C. E. and Bhagwat P., "Highly dynamic Destination- Sequenced Distance-Vector routing (DSDV) for mobile computers", In proceedings of SIGCOMM '94 Conference on Communications Architectures, Protocols and Applications. 1994, pp.234-244.

[4] Murthy C. S. R. and Manoj B. S., "Ad Hoc Wireless Networks: Architecture and Protocols", Pearson Education, 2005, pp. 330-334.

[5] Mahdipour E., Rahmani A. M. and Aminian E., "Performance Evaluation of Destination-Sequenced Distance-Vector (DSDV) Routing Protocol", In proceedings of IEEE International Conference on Future Networks, March 2009, pp. 186-190.

[6] Zheng Yihui, Que Xirong, Wang Wendong, Gong Xiangyang and Ma Jian, "N3S-OLSR: Node-Status SelfSensing Optimized Link-State Routing Protocols for MANET", In proceedings of IEEE Internaional Conference on Communications and Mobile Computing (CMC), 2010, pp. 288-292.
[7] Arora Deepali, Millman Eamon and Stephen W Neville, "Assessing the performance of AODV, DYMO and OLSR Routing Protocols in the Context of Larger-scale Denser MANETs", In proceedings of IEEE Pacific Rim Conference on Communications, Computers and Signal Processing (PacRim), 2011, PP. 675-679.

[8] Mohapatra S. and Kanungo P., "Performance Analysis of AODV, DSR, OLSR and DSDV Routing Protocols using NS2 Simulator", International Conference on Communication Technology and System Design, available online at www.sciencedirect.com, www.elsevier.com/locate/procedia, Procedia Engineering, 2012, pp.69-76.

[9] Rahman M. and Muktadir A. Al, "The impact of data send rate, node velocity and transmission range on QoS parameters of OLSR and DYMO MANET routing protocols", In proceedings of IEEE 10th international Conference on Computer and Information Technology, ICCIT, Dec. 2007. pp. 1-6.

[10] The network simulator - ns-2, http://www.isi.edu/nsnam/ns/on. The CMU Monarch Project, Computer Science Department, Carnegie Mellon University, //www.cs.cmu.edu. , Accessed in Dec. 2013.

[11] Francisco J. Ros, "um-olsr-1.0.tgz", University of Murcia, Spain, available online at http://masimum.inf.um.es/ fjrm/development/um-olsr/ and http://sourceforge.net/ projects/um-olsr/, 2006. Accessed in Jan. 2015.

[12] Kumawat Ramprasad and Somani Vinay, "Comparative Analysis of DSDV and OLSR Routing Protocols in MANET at Different Traffic Load", In proceedings published by International Journal of Computer Applications-International Conference on Computer Communication and Networks (CSI-COMNET), 2011, pp. 34-39.

[13] Qayyum A., Viennot L. and Laouiti A., "Multipoint Relaying for Flooding Broadcast Messages in Mobile Wireless Networks", In proceedings of IEEE 35th Annual Hawaii International Conference on System Sciences (HICSS), 2002, pp. 1-10.

[14] Abolhasan M., Wysocki A. and Dutkiewicz E., "A review of routing protocols for mobile ad hoc networks", Available online at www.elsevier.com/locate/ adhoc, Ad Hoc Networks, 2004, pp.1-22.

[15] Huhtonen Aleksandr, "Comparing AODV and OLSR Routing Protocols", Telecommunication Software and Multimedia Laboratory. HUT T-110.551 Seminar on Internetworking, Sjokulla, available online at www.tml.tkk.fi/ Studies/T- 110.551/2004/ papers/ Huhtonen.pdf, 2004, pp. 1-9.

[16] Dhurandher S. K., Obaidat M. S. and Gupta M., "A reactive Optimized Link State Routing protocol for Mobile ad hoc networks", In proceedings of 17th IEEE International Conference on Electronics, Circuits, and Systems, 2010, pp. 367-370.

[17] Royer E. M. and Toh C. K., "A Review of Current Routing Protocols for ad hoc mobile Wireless Networks", In IEEE Personal Communications Magazine, April 1999, pp. 46-55.

[18] Salman Javed, "Performance Analysis of OLSR Protocol in a Mobile a Mobile Ad Hoc Wireless Network", In proceedings of IEEE $2^{\text {nd }}$ International Conference on Computer, Control \& Communication, Feb. 2009, pp. 1-5.

[19] Shakkeera, "Optimal path selection technique for flooding in link state routing protocol using forwarding mechanisms in MANET", In proceedings of IEEE International Conference on Communication and 
Computational Intelligence (INCOCCI), Dec. 2010, pp. 318-323.

[20] Huang Y., Bhatti S. and Parker D., "Tuning OLSR", In proceedings of $17^{\text {th }}$ Annual IEEE International Symposium on Personal, Indoor and Mobile Radio Communications, Nov. 2006, pp. 1-5.

[21] Yamada Kenji, Itokawa Tsuyoshi and Kitasuka, Aritsugi Masayoshi, "Cooperative MPR Selection to Reduce Topology Control Packets in OLSR", In proceedings of IEEE International Conference-TENCON, 2010, pp. 293298.

[22] Ying Ge, Thomas Kunz and Louise Lamont, "Quality of Service Routing in Ad-Hoc Networks Using OLSR", In Proceeding of the IEEE 36th Hawaii International Conference on System Science (HICSS), 2003, pp. 1-9.

[23] Shahram Behzad, Reza Fotohi and Shahram Jamali, "Improvement over the OLSR Routing Protocol in Mobile Ad Hoc Networks by Eliminating the Unnecessary Loops", Published online in MECS I.J. of Information Technology and Computer Science, DOI: 10.5815/ijitcs.2013.06.03, May 2013, pp. 16-22.

[24] Dilpreet Kaur and Naresh Kumar, "Comparative Analysis of AODV, OLSR, TORA, DSR and DSDV Routing Protocols in Mobile Ad-Hoc Networks", Published online in MECS I.J. of Computer Network and Information Security, DOI: 10.5815/ijcnis.2013.03.05, March 2013, pp. 39-46.

\section{Authors' Profiles}

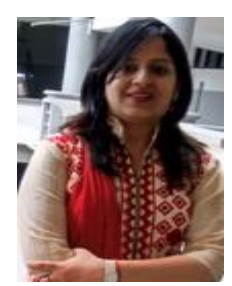

Shelja Sharma: Currently pursuing Doctoral Research in the area "Mobile Ad Hoc Networks" from Manav Rachna International University, Faridabad, India. She has received Bachelor's of Engineering degree in Computer Science \& Engineering with Honors from Rajasthan University, Jaipur, India in 2005 and Masters of Technology degree in Computer Engineering from Manav Rachna International University, Faridabad, India in 2011.

Presently she is working as an ASSISTANT PROFESSOR in the Department of Computer Science \& Engineering, Faculty of Engineering \& Technology, Manav Rachna International University, Faridabad, India. She has 9 years of total experience and published 6 Research publications in International Journals and IEEE International conferences. Her primary research area includes "Mobile Ad Hoc Networks".

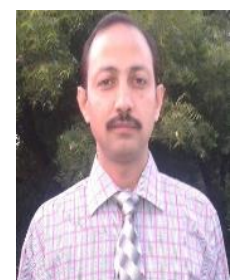

Dr. Suresh Kumar Bhardwaj: Currently working as a PROFESSOR, in the Department of Computer Science \& Engineering, Faculty of Engineering \& Technology, Manav Rachna International University, Faridabad, India. He has more than 14 years of experience and more than 30 research publications in diversified areas. His areas of interest are Ad hoc Networks, Database Management Systems and Data Mining.

How to cite this paper: Shelja Sharma, Suresh Kumar Bhardwaj,"Experimental Analysis of OLSR and DSDV Protocols on NS-2.35 in Mobile Ad-Hoc Networks", IJCNIS, vol.7, no.8, pp.21-29, 2015.DOI: 10.5815/ijcnis.2015.08.03 